\section{A Universal Ankle-Foot Prosthesis Emulator for Human Locomotion Experiments}

\author{
Joshua M. Caputo \\ Experimental Biomechatronics Laboratory, \\ Department of Mechanical Engineering, \\ Carnegie Mellon University, \\ Pittsburgh, PA 15213 \\ e-mail: jmcaputo@andrew.cmu.edu

\section{Steven H. Collins ${ }^{1}$} \\ Experimental Biomechatronics Laboratory, \\ Department of Mechanical \\ Engineering \& Robotics Institute, \\ Carnegie Mellon University, \\ Pittsburgh, PA 15213 \\ e-mail: stevecollins@cmu.edu
}

Robotic prostheses have the potential to significantly improve mobility for people with lower-limb amputation. Humans exhibit complex responses to mechanical interactions with these devices, however, and computational models are not yet able to predict such responses meaningfully. Experiments therefore play a critical role in development, but have been limited by the use of productlike prototypes, each requiring years of development and specialized for a narrow range of functions. Here we describe a robotic ankle-foot prosthesis system that enables rapid exploration of a wide range of dynamical behaviors in experiments with human subjects. This emulator comprises powerful off-board motor and control hardware, a flexible Bowden cable tether, and a lightweight instrumented prosthesis, resulting in a combination of low mass worn by the human $(0.96 \mathrm{~kg})$ and high mechatronic performance compared to prior platforms. Benchtop tests demonstrated closedloop torque bandwidth of $17 \mathrm{~Hz}$, peak torque of $175 \mathrm{Nm}$, and peak power of $1.0 \mathrm{~kW}$. Tests with an anthropomorphic pendulum "leg" demonstrated low interference from the tether, less than $1 \mathrm{Nm}$ about the hip. This combination of low worn mass, high bandwidth, high torque, and unrestricted movement makes the platform exceptionally versatile. To demonstrate suitability for human experiments, we performed preliminary tests in which a subject with unilateral transtibial amputation walked on a treadmill at $1.25 \mathrm{~ms}^{-1}$ while the prosthesis behaved in various ways. These tests revealed low torque tracking error (RMS error of $2.8 \mathrm{Nm}$ ) and the capacity to systematically vary work production or absorption across a broad range (from -5 to $21 \mathrm{~J}$ per step). These results support the use of robotic emulators during early stage assessment of proposed device functionalities and for scientific study of fundamental aspects of human-robot interaction. The design of simple, alternate end-effectors would enable studies at other joints or with additional degrees of freedom. [DOI: 10.1115/1.4026225]

Keywords: prosthetic foot, design, biomechanics, walking, amputee, gait

\section{Introduction}

Individuals with lower-limb disabilities experience reduced mobility and quality of life compared to their able-bodied

\footnotetext{
${ }^{1}$ Corresponding author.

Contributed by the Bioengineering Division of ASME for publication in the Journal of Biomechanical Engineering. Manuscript received February 22, 2013; final manuscript received December 4, 2013; accepted manuscript posted December 12, 2013; published online February 13, 2014. Assoc. Editor: Kenneth Fischer.
}

counterparts. Major lower-limb amputation is a prominent example, affecting more than 600,000 people in the United States, disproportionately affecting disadvantaged groups, and expected to double in prevalence by the year 2050 [1]. Individuals with amputation experience decreased walking performance using conventional, passive prostheses, including increased metabolic energy consumption, slower preferred walking speed, increased likelihood of falling, increased loading and injury of the unimpaired limb, and restricted social and recreational engagement [2-13].

Robotic devices with active assistance show promise for improving locomotor performance for people with lower-limb amputation [13-19]. For example, the first robotic ankle-foot prosthesis to reduce the energetic cost of walking for amputees has recently been demonstrated [20]. A similar function can be provided by recycling energy that would otherwise be dissipated, suggesting that this benefit might be obtained in designs without motors or batteries [21]. These results highlight the potential for positive impact through robotic technology development.

Advances in robotic ankle prosthesis technology have been achieved despite very limited exploration of possible functionalities. A primary goal is to improve locomotor performance, which is a deceptively challenging task [22]. Human adaptations to mechanical interactions with a prosthesis are very difficult to predict. Although computational models can predict some qualitative aspects of human walking [23,24], and underlying control [25], they are not yet capable of predicting responses to subtle mechanical changes $[26,27]$. Experimental studies are therefore crucial to evaluating the effects of a proposed design on humans, but such tests require a physical device that can be worn and used by a person. This has led to the development of product-like prototypes, each embodying a candidate functionality (we use this term in the manner of [28]), and each requiring several years of design and refinement prior to evaluation by human users [14-16,20,21,29]. Autonomy presents the greatest design challenge, leading to specialized devices that are not versatile enough to express other candidate functionalities. This limits their usefulness as experimental tools and prevents studies with broadly generalizable findings; even in the cases where results have been positive, we have not understood why or whether better solutions exist. Our field has thereby invested heavily in answering "how" to implement various functionalities, while the more important question of "what" functionalities would most benefit the user remains largely unanswered. We propose that decoupling the critical task of testing proposed functionalities from the arduous task of designing specialized devices would speed the development of robotic prostheses with predictable benefits.

Laboratory testbeds, which have often been used as versatile exploratory tools in basic locomotion research, may provide the foundation for such an approach. These systems have typically been used as probes, providing measurable, though not tightly controlled, disturbances in experiments designed to gain insights into reflexes [30], adaptations to external [31] and internal [32] forces on the legs, adaptations to altered effects of muscle activity [33], and balance strategies [34]. This approach has cleverly leveraged machines with modest mechatronic performance to obtain useful insights. With improved fidelity, perhaps similar tools could be used to emulate behaviors relevant to specialized, wearable robots.

We propose that high-performance testbeds, or "emulators," could be used to explore potential robotic assistance strategies, allowing measurement of human responses without the timeconsuming development of specialized prototypes. Studies could be conducted during early product development, emulating, testing, and refining proposed product designs quickly and with low cost. Emulators could also facilitate scientific investigations that address fundamental aspects of human-robot interaction during biomechanics tasks. This approach seems to have been first suggested by [35] in the context of prosthetic knees, and later extended to prosthetic elbows by [36]. 


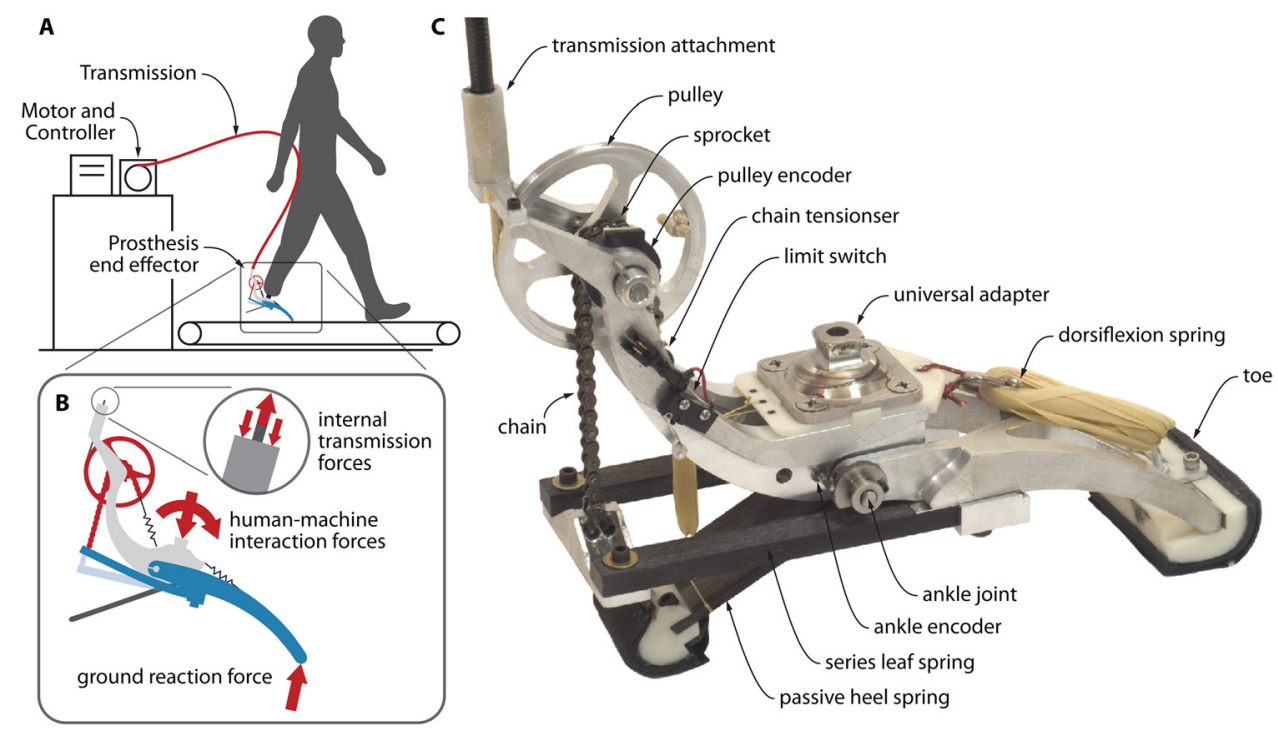

Fig. 1 Mechatronic design of the universal prosthesis emulator. (a) The system comprises three elements: (1) powerful off-board motor and control hardware, (2) a flexible tether transmitting mechanical power and sensor signals, and (3) a lightweight instrumented end-effector. This division of components was chosen to maximize responsiveness and minimize endeffector mass during treadmill walking. (b) Free-body diagram of the end-effector. Internal Bowden cable transmission forces pull the synthetic rope upwards while equally and oppositely pushing the aluminum frame downwards. Rope tension is transmitted through the pulley, sprocket, chain, and leaf spring, giving rise to a ground reaction force at the toe. The effect is equivalent to an ankle plantarflexion torque, resulting in a reaction force and moment at the interface with the human user. (c) Photograph of the instrumented prosthesis. A pulleysprocket component magnifies transmission forces and allows direct measurement of spring deflection. A tensioning spring keeps the chain engaged. A limit switch protects against excessive plantarflexion. A universal adapter attaches to the socket or prosthesis simulator worn by the user. A dorsiflexion spring comprised of rubber bands retracts the toe, e.g., during leg swing. Fiberglass leaf springs provide series elasticity for ankle torque measurement and control. A separate leaf spring directly connected to the frame (not the toe) comprises the heel.

Experimental emulators for these purposes should provide versatile, high-performance control and actuation capabilities without restricting other natural motions of the body. Torque control allows the testbed to be used as a haptic interface [37], in which forces mimic the presence of a virtual system designed by the experimenter, such as springs or force fields. Torque control provides excellent versatility compared to, e.g., position control for which interactions are dominated by robot position, which can restrict human engagement [38]. Such a system should of course be capable of humanlike torque and power magnitudes, but the limiting factor that must be maximized for dynamic emulation is closed-loop torque bandwidth [39]. In this context, bandwidth is a measure of the responsiveness of the system, characterizing how quickly joint torque can be changed. High bandwidth might suggest large motors worn by the person, but such an approach would conflict with the requirement that interference with natural motions be minimized; mass worn on the limbs strongly affects coordination patterns and energetic cost [40]. Instead, emulators can leverage the advantages of a laboratory setting by placing motor and control components off-board and transmitting mechanical power through flexible tethers to lightweight endeffectors worn on the body. This organization of components has the added advantage of allowing a single actuator and tether to control multiple swappable end-effectors, with each being significantly simpler to design and fabricate than a fully integrated system. External forces from tethers could also interfere with natural motions of the limbs [31,41], so tethers should be designed to be lightweight and flexible, and their effects on gait should be measured and minimized.

Here we describe the design and feasibility tests of one such emulator for ankle-foot prostheses. We chose the ankle for its commonality to lower-limb disabilities [7] and mechanical importance in locomotion [42]. We used an off-board motor tethered to a lightweight prosthesis through a Bowden cable. We performed a variety of tests, in isolation and during human walking, to characterize performance and suitability of the platform as an experimental tool.

\section{Methods}

We designed and constructed a tethered ankle-foot prosthesis system incorporating a powerful electric motor, a lowinterference transmission, and a lightweight instrumented prosthesis. We implemented plantarflexion torque control at the prosthetic ankle joint, including a demonstration mode suitable for walking tests, and measured system performance in a series of benchtop tests and human walking trials.

2.1 Mechatronic Design. The electromechanical system comprises an off-board motor and control system, a flexible tether, and an instrumented prosthesis end-effector (Fig. 1). We selected a powerful, low inertia electric motor and a high-speed, real-time control module for off-board actuation. We used a $1.61 \mathrm{~kW} \mathrm{AC}$ servomotor with a 5:1 planetary gearhead (BSM90N-175AF with GBSM90-MRP120-5, Baldor Electric Corp., Fort Smith, AR). We made this selection using computer simulations of closed-loop torque control characteristics in which inertia, motor constant, and gear ratio were varied and step response and bandwidth were estimated. We regulated motor voltage using an industrial motor drive (MFE460A010B, Baldor) with embedded velocity control. Desired motor velocity commands were generated using a real-time controller (ACE1103, dSPACE Inc., Wixom, MI) based on high-level control laws (see Sec. 2.2) and were communicated on an analog channel at $500 \mathrm{~Hz}$. We used a Bowden cable transmission 
comprised of a coiled-steel outer conduit (415310-00, Lexco Cable Mfg., Norridge, IL) and a $3 \mathrm{~mm}$ synthetic inner rope (Vectran Fiber Inc., Fort Mill, SC). The cable measured $3.5 \mathrm{~m}$ in length and was routed to minimize bending, thereby minimizing friction while allowing desired end-effector motions [Fig. 1(a)]. We fixed the outer conduit to the motor frame on one end and to the prosthesis frame on the other end, then wrapped the inner rope onto the motor pulley on one end and onto the prosthesis pulley on the other end [Fig. 1(c)]. Forces generated by the motor were thereby transmitted to the prosthesis independent of its position in the workspace. Sensor cables were bundled with the Bowden cable to complete the tether.

We designed an instrumented prosthesis end-effector to convert transmission forces into ankle plantarflexion torques. The prosthetic ankle joint [Fig. 1(c)] allows the toe segment to rotate with respect to the prosthesis frame. A series leaf spring on the toe segment protrudes backwards relative to the ankle joint axis. Transmission forces pull upwards on the end of this spring, generating a plantarflexion moment about the ankle joint [Fig. 1(b)], similar to the action of the Achilles tendon in the human ankle. We included a series spring to decouple motor inertia from the toe segment, which can improve torque tracking during, e.g., intermittent ground contact $[43,44]$. We also used spring deflection to measure ankle torque based on a calibrated model (see Sec. 2.2). We used a low-tension spring to pull the toe upwards, leading to ankle dorsiflexion when transmission forces were low. We directly attached a compliant heel segment to the prosthesis frame.

Prosthesis dimensions were selected based on those of an average human foot [45]. The distance between the heel and the toe was $0.22 \mathrm{~m}$. The foot was aligned on the user's leg such that the heel was $0.07 \mathrm{~m}$ to the rear of the centerline of the tibia or pylon. The ankle was $0.07 \mathrm{~m}$ from the ground plane during standing. The toe was $0.07 \mathrm{~m}$ wide and the heel was $0.04 \mathrm{~m}$ wide, slightly narrower than the typical human dimensions of 0.10 and $0.07 \mathrm{~m}$, respectively. This allowed for a variety of foot widths to be explored using toe and heel attachments. Although the prosthesis fit inside an unmodified shoe, interference with the series leaf springs prevented its use during the tests reported here. Instead, rubber at the toe and heel contact points approximated the effects of the sole of a walking shoe.

We constructed the prosthesis end-effector using a variety of custom and catalog components. Series leaf springs and heel springs were machined from fiberglass (unidirectional E-Glass, GC-67-UB, Gordon Composites Inc., Montrose, CO). Heel and toe pads were 3D printed using fused deposition modeling (FDM) of acrylonitrile butadiene styrene (ABS). Rubber strips (Pro Tania, Vibram, North Brookfield, MA) were affixed with adhesive at ground contact locations. The frame, toe segment, spring attachment, pulley, and Bowden cable termination components were machined from 7075-T651 aluminum. We connected the pulley and series springs with an ANSI 25 hardened steel sprocket and roller chain (6Q 7-H25 and A 6C 7-25011C, Stock Drive Products/Sterling Instrument, New Hyde Park, NY) including a custom machined 1074/1075 spring steel link at the spring attachment. The device was connected to the user's pylon with a standard titanium prosthesis adapter (FND-227014, Ohio Willow Wood, Sterling, OH). We measured ankle rotation with a 10 bit absolute magnetic encoder with analog transmission, geared 8:1 for increased resolution, and pulley rotation with a 9 bit optical incremental encoder (MAE3-A10-250-220-7-1 and E8P-512-250D-H-D-2, respectively, US Digital, Vancouver, WA). See Supporting Materials for models of all custom components and a complete bill of materials.

2.2 Sensing and Control. We computed measured ankle torque based on measurements of spring displacement and ankle position using a calibrated model. We performed calibration trials in which the prosthesis was fixed upside down while masses of known weight were hung from the toe. We applied a range of masses and ankle angles that spanned the expected operating conditions. Maximum torque during calibration was limited by the rated continuous current of the motor (which is lower than the maximum intermittent current). We modeled measured ankle torque as a function of ankle angle and prosthesis pulley angle, and fit coefficients using least squares regression. We first determined a relationship between ankle and pulley angles under zero load, then determined a stiffness coefficient for the deviation of the pulley angle from the zero-torque relationship under various loads.

Torque control was achieved using proportional feedback on torque errors:

$$
\omega_{m}=K_{p}\left(\tau_{d}-\tau\right)
$$

where $\omega_{m}$ is the velocity commanded to the motor driver, $K_{p}$ is the proportional gain, and $\tau_{d}$ and $\tau$ are desired and measured ankle torque, respectively. $K_{p}$ was determined from a mathematical model, then hand tuned. We used a similar feedback control law to perform ankle position control under conditions with no external load, such as the swing phase of walking, by substituting a position error for torque error and using a modified gain. See Supporting Materials for a complete set of software used to control the device.

We designed several safety features, in both software and hardware, to limit the forces exerted by the prosthesis on the human user. We placed software limits on the maximum desired torque and motor velocity, and used software stops to prevent travel beyond the range of motion of the prosthesis ankle joint. We incorporated an electrical plantarflexion limit switch [Fig. 1(c)] and electrical buttons accessible to the subject and experimenter that deactivated the motor when pressed. Mechanical fail-safes included a transmission break-away, composed of an empirically determined number of loops of thin synthetic rope, and hard stops at the ankle's range of motion.

2.3 Benchtop Testing Methods. We conducted benchtop tests characterizing device performance in terms of torque measurement accuracy, peak torque, closed-loop torque step response, closed-loop torque bandwidth, peak power, and tether interference. These tests were designed to reveal fundamental aspects of system performance and to allow comparison with existing platforms.

We first evaluated the accuracy of our calibrated torque measurement. We applied a range of known ankle torques using static loading with free weights [Fig. 2(a)]. We separately applied each load with the ankle joint maximally dorsiflexed, in a neutral position, and maximally plantarflexed. We then compared measured and applied torques, computing the root mean square (RMS) error and the maximum absolute error. Validation and calibration were performed separately, comprising independent data sets.

We performed step response tests with the toe fixed in place to characterize closed-loop torque response time and demonstrate peak torque capacity. We rigidly fixed the prosthesis frame and toe to the benchtop, locking the ankle joint [Fig. 2(b)], and programmed desired torque as a square wave with a magnitude of $175 \mathrm{Nm}$. We then tuned $K_{p}$ so as to minimize rise time and overshoot. We collected data for 10 complete cycles, averaged the measured torque trajectories, and computed $90 \%$ rise and fall times.

We performed similar step response tests with a compliant load to demonstrate peak power. We rigidly fixed the prosthesis frame to the benchtop and attached the toe to the benchtop through a coil spring. We chose a spring with stiffness of $26,000 \mathrm{~N} \mathrm{~m}^{-1}$, which we found allowed motor velocity saturation. We collected data for 10 complete cycles, averaged the computed power trajectories, and computed peak power as the maximum of the average power trajectory.

We characterized closed-loop torque control bandwidth using frequency-domain transforms of the system's response to a chirp 
A Torque Measurement Accuracy

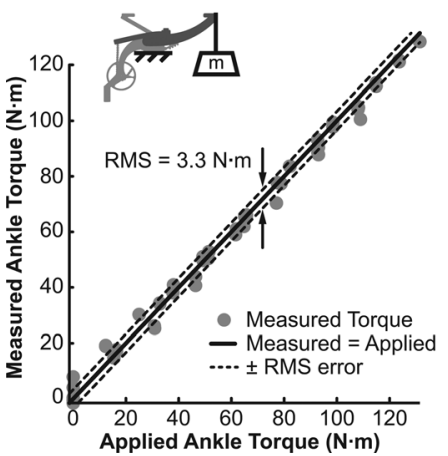

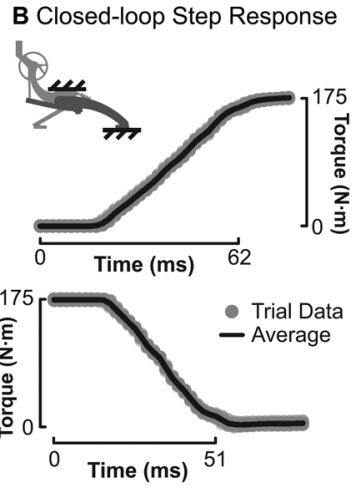

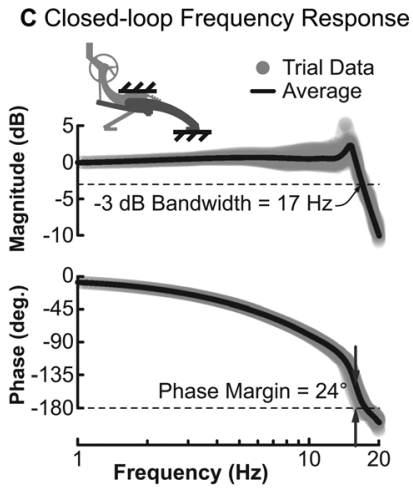

Fig. 2 Results of benchtop tests of mechatronic performance with the experimental prosthesis emulator. (a) Torque measurement accuracy. We performed tests in which we applied known torques by suspending weights from the toe in a range of known configurations, and found RMS measurement error of $3.3 \mathrm{~N} \mathrm{~m}$. (b) Closed-loop torque step response. We fixed the base and toe of the prosthesis and applied $175 \mathrm{Nm}$ step changes in desired torque. Across 10 trials, we measured average $90 \%$ rise times of $0.062 \mathrm{~s}$. (c) Bode plot of frequency response under closedloop torque control. We fixed the base and toe of the prosthesis and applied chirps in desired torque from 56.5 to $133 \mathrm{~N} \mathrm{~m}$, then smoothed the resulting curves and averaged over 10 trials. We calculated an average $-3 \mathrm{~dB}$ bandwidth of $17 \mathrm{~Hz}$.

in desired torque. During bandwidth trials, we rigidly fixed the prosthesis frame and toe to the benchtop, locking the ankle joint [Fig. 2(c)], and programmed desired torque as an offset chirp oscillating between 56.5 and $113 \mathrm{Nm}$ at frequencies rising from 0 to $30 \mathrm{~Hz}$. We tuned $K_{p}$ to maximize bandwidth with acceptable resonance. We mathematically approximated both input (desired torque) and output (measured torque) signals in the frequency domain using a fast Fourier transform (FFT). We calculated magnitude responses as the ratio of the magnitudes of the complex FFTs and frequency response as the difference of the angles of the complex FFTs. Accuracy of the FFTs obtained in each trial was limited by the number of data points captured at each input frequency, which was limited by trial duration prior to reaching motor temperature limits. We collected data for 10 trials, smoothed each resulting Bode plot to remove FFT artifacts, and averaged across trials. We calculated bandwidth as the minimum frequency for which the average magnitude response was above $-3 \mathrm{~dB}$. We calculated phase margin as the difference between $-180 \mathrm{deg}$ and the average phase response at the frequency where the magnitude response was $0 \mathrm{~dB}$.

We performed experiments with an anthropomorphic pendulum "leg" to characterize interference in natural leg motions due to tether stiffness and damping. In order to generate dynamic leglike motions under controlled, repeatable conditions, we constructed a single-link pendulum with mass properties of a 50th percentile male leg [46]. We attached the instrumented prosthesis to the end of this "leg," and performed trials under two conditions: tethered and untethered. In tethered, peak ankle plantarflexion torque was applied to maximize resistance to leg motions. For each trial, we raised the prosthesis to a consistent initial angle, allowed it to swing freely until a lower threshold amplitude was crossed, and recorded the number of cycles and the time elapsed. We conducted 10 trials and calculated the average frequency and decay time for each condition. We then calculated the stiffness and damping coefficient attributed to tether forces (see Appendix A for a detailed model and calculation).

2.4 Human Walking Testing Methods. We developed a high-level impedance control law that calculated desired torque based on ankle angle and gait cycle phase. This control law enabled evaluation of device performance during walking, and provided an example of one of many high-level torque control techniques that could be embodied by the system, such as impedance matching [16], proportional electromyography [41], positive force feedback [47], or time-trajectory control variants [48]. In the example control law (Fig. 3), desired torque was determined using the piecewise linear function

$$
\tau_{d}=k_{i}\left(\theta-\theta_{0_{i}}\right)
$$

where $\tau_{d}$ is desired ankle torque, $\theta$ is ankle joint angle, and $k_{i}$ and $\theta_{0_{i}}$ are the piecewise constant stiffness and offset terms, respectively, that remained constant over a range of joint angles and during each finite state $\phi$. A finite-state machine advanced $\phi$ through three phases: dorsiflexion, during the beginning of stance, characterized by negative ankle velocity; plantarflexion, during the end of stance, characterized by positive ankle velocity; and swing, characterized by no ground contact. During each of the stance phases, two values of $k_{i}$ and $\theta_{0_{i}}$ were used, based on a transition threshold value for $\theta$, such that the ankle joint behaved as a stiffening spring comprised of two linear stiffness regions. Different

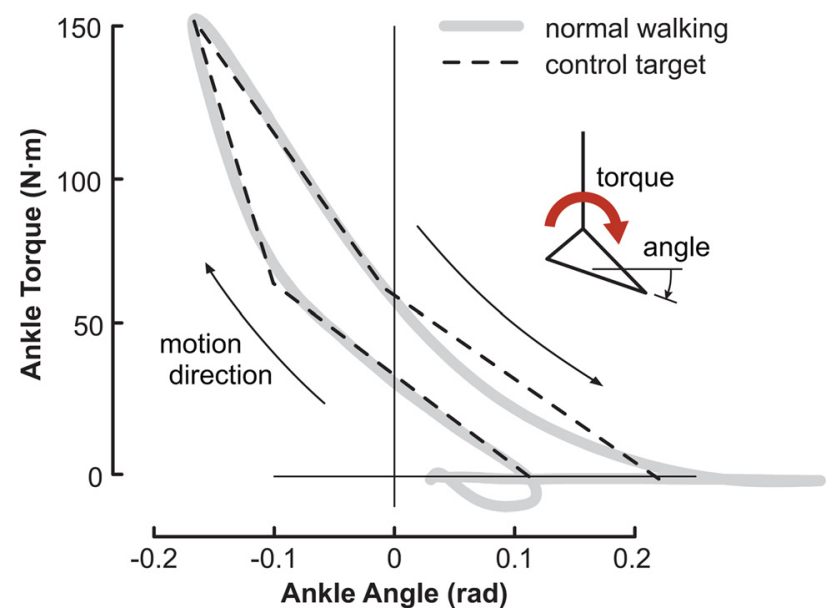

Fig. 3 Impedance control law used during walking trials. Desired torque is a piecewise linear function of ankle position, with separate dorsiflexion (negative velocity) and plantarflexion (positive velocity) phases. Default curve parameters were selected to roughly match the torque-angle relationship observed for the biological ankle during normal walking. Plantarflexion segments were manipulated across conditions to alter the net positive ankle joint work over the step cycle. 
values of $k_{i}$ and $\theta_{0_{i}}$ were used during dorsiflexion and plantarflexion phases, enabling control of the net work produced or absorbed over the course of a step cycle, equivalent to the area within the resulting work loop.

We chose default values of $k_{i}$ and $\theta_{0_{i}}$ for each linear segment such that the overall curve approximated the relationship observed for the human ankle during normal walking (Fig. 3). We also determined alternate sets of curve parameters for the plantarflexion phase that resulted in a range of values of net ankle work. During the swing phase, a separate position control mode reset the ankle angle to prepare for the next stance phase.

We used a configuration prediction term to improve tracking of desired torques generated using the impedance control law. We found that communication delays and motor dynamics typically led to a lag of about $16 \mathrm{~ms}$ between commanded and observed motor velocity changes. This caused measured torque to lag the impedance-based desired torque, especially during fast ankle motions, e.g., push off. We modified the ankle angle used to generate desired torque in Eq. (2) to account for expected changes in desired torque as follows:

$$
\theta_{p}=\theta+t_{\text {pred }} \dot{\theta}
$$

where $\theta_{p}$ is the predicted ankle angle substituted for $\theta$ in Eq. (2), $t_{\text {pred }}$ is a prediction time constant, and $\dot{\theta}$ is the current ankle angular velocity. This adjustment was based on a simplified model of the system dynamics, in which motor dynamics are much faster than ankle dynamics, and resulted in improved torque tracking. This is mathematically identical to including a derivative term in the desired torque calculation [Eq. (2)].

We performed a series of walking tests to evaluate torque tracking performance and demonstrate system versatility under realistic operating conditions. One subject with unilateral transtibial amputation (male, $88 \mathrm{~kg}, 0.92 \mathrm{~m}$ greater trochanter height, 44 years) wore the instrumented prosthesis while walking on a treadmill at a speed of $1.25 \mathrm{~ms}^{-1}$. Five conditions were applied, in which the prosthesis followed the impedance control law with conditionspecific plantarflexion parameters $k$ and $\theta_{0}$ corresponding to net work values of roughly $-1,0,1,2$, and 3 times the normal net work observed during walking. The subject walked for $7 \mathrm{~min}$ under each condition. Data from the final minute of each trial (about 50 prosthesis steps) were captured and normalized to percent stance (scaled time). For each condition, we calculated RMS error between desired and measured torque and the average and standard deviation of net ankle work per step.

\section{Results}

The instrumented prosthesis had a mass of $0.96 \mathrm{~kg}$ (weighed without the tether). The ankle range of motion was $14 \mathrm{deg}$ $(17 \mathrm{deg})$ in dorsiflexion and $35 \mathrm{deg}(27 \mathrm{deg})$ in plantarflexion when unloaded (maximally loaded). Torque measurement errors were always less than $7.9 \mathrm{Nm}$, with $3.3 \mathrm{Nm}$ RMS error (or $1.9 \%$ of maximum torque) [Fig. 2(a)]. Peak operating torque was demonstrated to be at least $175 \mathrm{Nm}$ [Fig. 2(b)].

Peak ankle power output was $1036 \pm 44 \mathrm{~W}$ (mean \pm st. dev.), with a corresponding ankle torque of $144 \pm 1 \mathrm{~N} \mathrm{~m}$ and velocity of $7.2 \pm 0.3 \mathrm{rads}^{-1}$. At the instant of peak ankle power output, both the series spring and tether were being stretched (absorbing energy) and therefore did not contribute to peak power, e.g., through oscillations. The motor reached velocity saturation during each peak power trial.

We measured closed-loop ankle torque step response rise times ( $90 \%$ of final value) to be $0.062 \pm 0.000$ and $0.051 \pm 0.001 \mathrm{~s}$ for increasing and decreasing steps, respectively [Fig. 2(b)]. We calculated closed-loop ankle torque response to have a bandwidth ( $-3 \mathrm{~dB}$ magnitude criteria) of $17.1 \pm 0.2 \mathrm{~Hz}$ and a phase margin of $23.6 \pm 5.3 \mathrm{deg}$ [Fig. 2(c)]. We found that increasing $K_{p}$ from the tuned value resulted in resonance at about $15 \mathrm{~Hz}$.

In experiments with the anthropomorphic pendulum leg, we characterized tether interference as a rotational stiffness $k_{t}$ and damping $b_{t}$ about the hip joint. We found very low stiffness and damping, with $k_{t}=2.6 \pm 0.11 \mathrm{~N} \mathrm{~m} \mathrm{rad}^{-1}$ and $b_{t}=0.26 \pm 0.023$ $\mathrm{Nm}\left(\mathrm{rad} \mathrm{s}^{-1}\right)^{-1}$. For comparison, the damping coefficient for untethered trials $b_{0}$ attributable to the ball bearing and air resistance, was calculated to be $0.12 \pm 0.002 \mathrm{~N} \mathrm{~m}\left(\mathrm{rad} \mathrm{s}^{-1}\right)^{-1}$. The calculated tether stiffness and damping would result in an estimated $1 \mathrm{Nm}$ resistance torque at maximum hip flexion and $1 \mathrm{Nm}$ (or $1.9 \%$ maximum torque) at maximum hip velocity, under maximum transmission loads (i.e., peak ankle torque, the worst case). See Appendix A for detailed calculations.

During walking trials, measured torque closely matched desired torque for a variety of control parameters. In the condition corresponding most closely to normal ankle function, RMS torque error over time was $2.8 \mathrm{Nm}$, or about $2 \%$ of peak torque [Fig. 4(b)], characterizing temporal tracking performance. Torque tracking in joint angle space resulted in net ankle work production of $7.88 \pm 1.28 \mathrm{~J}$ [Fig. 4(a)]. Variability in work production was primarily due to natural variations in subject kinematics from step to step, evidenced by a similar standard deviation in desired work $(1.08 \mathrm{~J})$. The average work error was $-1.61 \mathrm{~J}$, due predominantly to tracking errors during rapid motions at terminal stance. The
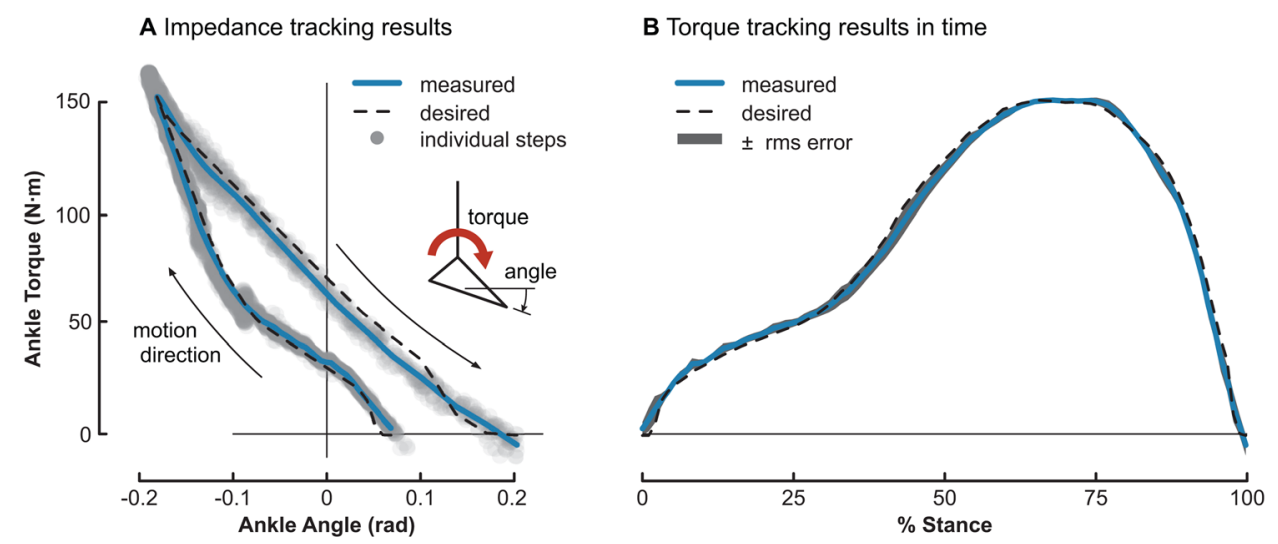

Fig. 4 Tracking of impedance control law during walking. (a) Measured torque-angle relationship as one subject with unilateral transtibial amputation walked at $1.25 \mathrm{~ms}^{-1}$ for $1 \mathrm{~min}(52$ strides). Each step resulted in a similar amount of net joint work, $7.88 \pm 1.28 \mathrm{~J}$, visible here as work-loop area. (b) Joint torque over the stance period during $1 \mathrm{~min}$ of walking, normalized to \% stance. Average stance duration was $0.58 \pm 0.02 \mathrm{~s}$. The average RMS torque error was $2.8 \mathrm{Nm}$. Note that time-trajectory error appears smaller than error in angle-torque space, while the latter is more meaningful in terms of work production or absorption. 


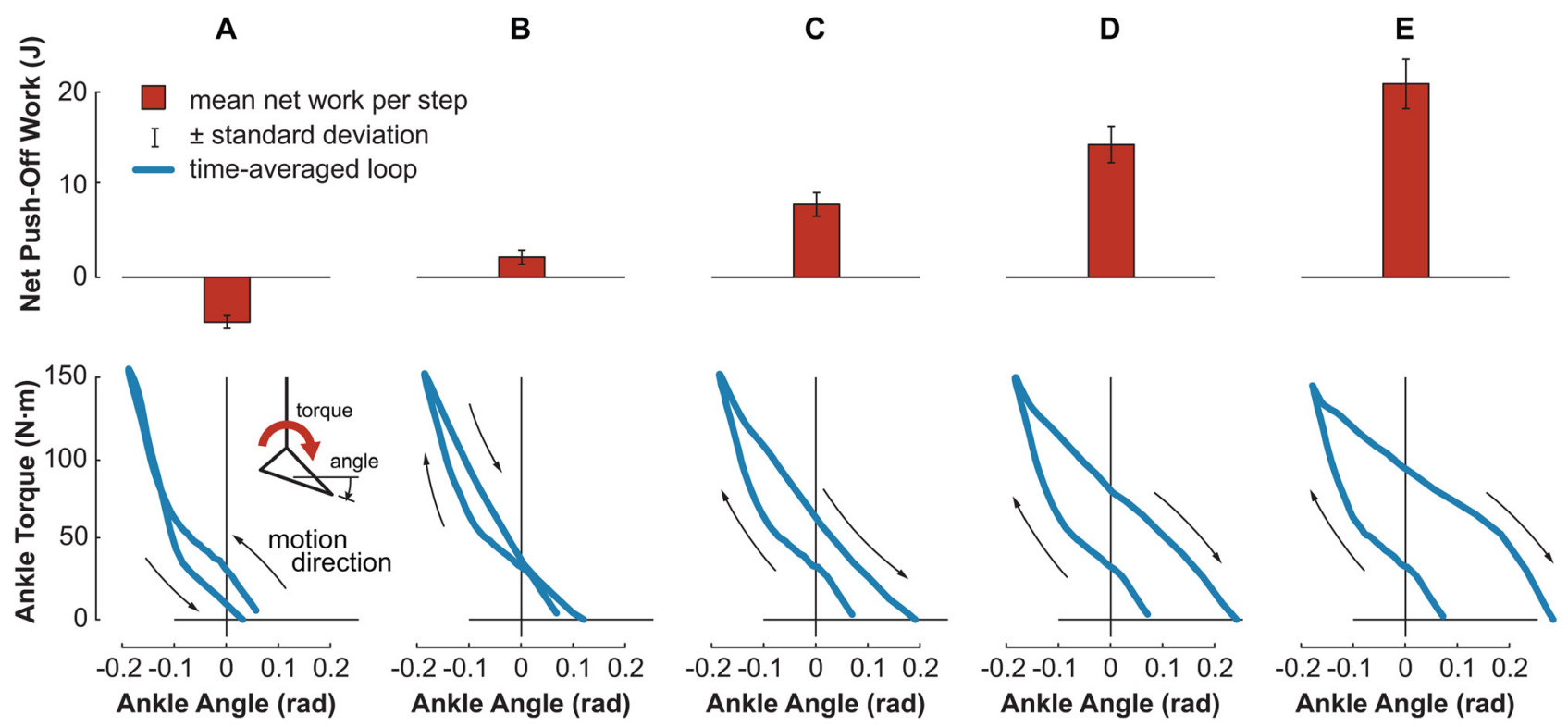

Fig. 5 Modulating the impedance control law parameters resulted in a variety of work loops during walking, demonstrating system versatility. We measured average net work per step as one subject with unilateral transtibial amputation walked at $1.25 \mathrm{~ms}^{-1}$ for $1 \mathrm{~min}$ (52 strides) with plantarflexion curve parameters set to five different values (a)-(e). Top: Average net joint work produced (positive) or absorbed (negative) during each step, mean \pm st. dev. Bottom: Average impedance relationship for each condition, computed as the time-averaged ankle torque by time-averaged joint angle. We measured energy absorption of $-5 \mathrm{~J}$ in condition (a), similar to the damping effects of conventional dynamic-elastic response prostheses. In condition (c), $8 \mathrm{~J}$ of work was produced per step, similar to the contribution of ankle plantarflexor muscles during human walking. We measured energy production of $21 \mathrm{~J}$ in condition (e), which would constitute a very large input from a robotic prosthesis.

standard deviation of error in mechanical work was $0.48 \mathrm{~J}$, or about $6 \%$ of the net work, characterizing the consistency of dynamical emulation. In trials with systematic variations in the control law (Fig. 5), we measured net ankle joint work values of $-4.8 \pm 0.7,2.2 \pm 0.8,7.9 \pm 1.3,14.4 \pm 1.9$, and $20.9 \pm 2.6 \mathrm{~J}$, again including variability due to natural variations in human stride kinematics. Average stance duration was $0.58 \pm 0.02 \mathrm{~s}$ and average stride period was $1.15 \pm 0.05 \mathrm{~s}$. These results demonstrate incremental improvements on results from earlier walking trials with an able-bodied subject wearing a simulator boot [49].

\section{Discussion}

We developed an experimental platform for use in early stage assessment of robotic ankle-foot prosthesis design concepts and conducted thorough tests of the system's mechatronic performance. Walking trials demonstrated precise torque tracking, both in time and in joint angle space, and versatile mechanical behavior through systematic changes in high-level control law parameters. Benchtop tests revealed superior performance compared to prior torque-controlled devices, particularly in terms of worn mass and torque bandwidth. These results suggest tethered robotic prostheses could be used to emulate and evaluate novel functionalities as part of an experimental approach to device development.

Pilot tests of walking with the prosthesis demonstrated the suitability of this experimental tool for emulating a wide variety of functionalities under realistic conditions. We measured very low torque tracking errors in time and in torque-angle space (Fig. 4), and found that work production could be systematically and consistently altered across conditions (Fig. 5). Prosthesis energy contributions are strongly involved in human performance [20,21], and affect key device design requirements, such as motor, battery, or spring size. Consistent work production is challenging in torque-controlled actuator systems, however, because small changes in the timing of torque production can result in significant changes in mechanical power. For instance, the push-off phase of gait occurs very rapidly [Fig. 4(b), 75\%-100\% stance] and is characterized by a large ankle plantarflexion velocity and a large drop in ankle torque. Leading or lagging the desired torque alters the resulting torque-velocity relationship, causing large changes in power and work. Most developers of lower-limb devices with torque control have not reported these errors. We demonstrated this system's capacity to systematically manipulate the torquedisplacement relationship during this phase of gait, leading to a range of overall ankle behaviors consistent with damped springs, passive springs, human ankle musculature, or high-powered robotic devices [Figs. 5(a)-5(e)]. These effects were highly repeatable, characterized by small standard deviation in prosthesis work error. Dynamic consistencies were not due to fixed features of the mechanical structure, such as parallel springs or dampers, which would limit versatility. This system can therefore emulate prosthesis designs with a wide range of mechanical features, and can even alter these features online, e.g., to optimize device performance for an individual user. Controlled step-by-step changes could also be used to address a variety of scientific questions, allowing direct measurement of human response to systematic changes in, e.g., dynamic stability [50] or altered metabolic cost landscapes [51,52].

The versatility observed during walking trials was achieved by leveraging the laboratory setting to improve mechatronic performance, particularly in terms of worn mass and closed-loop torque bandwidth. High closed-loop torque bandwidth is important for dynamic emulation during periods of rapidly changing conditions, such as the initial contact of the foot with the ground [53], while low mass is needed to avoid affecting natural limb motions or increasing user effort [54]. The prosthesis end-effector had lower mass than the lightest reported for comparable designs (0.96 versus $1.37 \mathrm{~kg}$ in [41]), yet with an order of magnitude greater bandwidth. Benchtop tests demonstrated higher closed-loop torque bandwidth than the highest open-loop bandwidth values reported for prior designs (17 versus $14 \mathrm{~Hz}$ in [14]), but with less than half the mass. The emulator also exhibited higher peak torque (175 versus $134 \mathrm{~N} \mathrm{~m}$ in [14]) and peak power (1036 versus $270 \mathrm{~W}$ 
in [55]) than prior experimental results. These results also compare well with observations of the human ankle and foot. We demonstrated peak torques $50 \%$ greater than those observed during human walking (1.6 $\mathrm{N} \mathrm{m} \mathrm{kg}^{-1}$ in [42]), device mass less than a human foot (1.5\% body mass in [46]), and torque bandwidth twice that of ankle muscles $(6-10 \mathrm{~Hz}$ in $[56,57])$. Some other actuators have demonstrated similar torque bandwidth, but with substantially lower peak torque and greater mass [29,32,58,59]. While adjustments to the features of other designs could result in improvements in one or another category of performance, overall performance differences seem to be related to qualitative differences in system design.

The primary feature allowing for improved mechatronic performance in this emulator was a Bowden cable tether separating the prosthesis end-effector from driving hardware. This division of components allowed the use of a powerful but heavy gearmotor $(1.61 \mathrm{~kW}, 16.4 \mathrm{~kg})$ without additional mass worn by the user. The tether itself did exert measurable forces on the prosthesis as it moved in the treadmill workspace, but tests with the anthropomorphic pendulum "leg" demonstrated these were minor compared to the forces typically measured in the leg during human locomotion. We estimated that, under worst-case assumptions, the tether would produce effective hip torques of at most $1 \mathrm{Nm}$, or about $1.9 \%$ of the peak torque attributed to hip muscles during normal walking [60]. By contrast, an additional $2 \mathrm{~kg}$ in end-effector mass due to a smaller actuator [61] would increase peak hip torque by about $18 \mathrm{Nm}$, or $48 \%$ [40], and metabolic cost by at least $17 \%$ [40] or as much as $48 \%$ [26]. The tether does, however, impose a restriction on the size of the workspace, and this limitation must be weighed against such benefits. Overground walking would be enabled by a longer tether with serpentine routing, but this would result in poorer mechatronic performance due to increased cable compliance and friction. Overground walking might also be achieved by, e.g., mounting the motor on a moving platform, but at a cost of greatly increased system complexity. It is therefore likely impractical to use the device for overground tests, restricting it to use in activities such as treadmill walking, stairmill climbing, and standing. This restriction does not interfere with measurement of common steady-state outcomes such as energy economy or maximum speed, but would interfere with tests of intrinsically overground activities. In a laboratory setting, replacing on-board hardware with a Bowden cable tethered to more capable off-board hardware is therefore advantageous in terms of increased versatility and decreased interference with natural human motions, but restricts the types of activities that can be tested.

For this type of application, Bowden cable tethers appear to have advantages over hydraulic or pneumatic transmissions in terms of mass, bandwidth, and interference with natural motions. Other designs utilizing Bowden cables have demonstrated strong mechatronic performance in their domains, such as ankle perturbation [30], low-interference knee actuation [32], or upperextremity force feedback [62]. Tethered hydraulic systems have demonstrated high torque bandwidth, but have been limited by the need for heavy cylinders at the end-effector [63] and relatively stiff, heavy hoses [64]. Transmission tubes in conventional pneumatic systems are often more flexible, as they are operated at lower pressure, but heavy cylinders are still needed [58] and gas compressibility limits bandwidth. Artificial pneumatic muscles can result in low end-effector mass [41], but have more severe bandwidth limitations due to increased resting volume $[65,66]$ and are still heavier than a Bowden cable termination. Like conventional hydraulic or pneumatic systems, Bowden cables can introduce stick-slip dynamics, due to cable-conduit or piston-cylinder friction, but these can be remedied by measuring torque on the joint side of the transmission [67]. Anecdotally, we found the Vectran cable used here to be quite durable (more than $10^{6}$ cycles), flexible (minimum bending radius $\approx 3$ cable radii), and tidy (no oil leaks, for example). We also found Vectran to be more robust than any of the steel wire rope constructions we tried.
In tethered applications where mass and torque bandwidth are critical, a synthetic Bowden cable architecture is likely to be advantageous.

Fiberglass leaf springs also contributed to low end-effector mass in this emulator. Physical series elasticity can reduce torque errors at instants of large position disturbance [43] such as the instant when ground contact is initiated at the beginning of the stance phase of gait [Fig. 4(a)]. However, these have often been designed as steel coil springs in compression $[29,61]$ or torsion [32], which can result in designs with greater mass than necessary. Fiberglass is eight times lighter than spring steel for a given strain energy capacity ( $\rho E \sigma_{y}^{-2}$, see Appendix B for derivation). This benefit is offset by spring geometry, since conventional fiberglass manufacturing limits spring shapes to simple beams, which are three times heavier than coil springs for a given energy capacity. Coil springs require additional structures to convert axial spring forces into joint torques, a function implicitly satisfied by the leaf spring itself, and the added mass of these structures can be approximated as that of the leaf spring for purposes of comparison. The use of fiberglass leaf springs can therefore reduce spring mass by about $70 \%$ compared to steel coil springs, and saved an estimated $0.19 \mathrm{~kg}$, or $20 \%$ end-effector mass, in this application.

Numerous aspects of this experimental emulator prototype could be improved to enhance overall performance. System responsiveness (bandwidth) was limited by peak motor velocity, which could be at least doubled for the same motor by the use of a power supply with higher voltage. Torque control could also be improved by the use of more sophisticated low-level programming than the proportional control scheme used here [Eq. (1)]. The Bowden cable used here exhibited significant stick-slip dynamics, and reducing conduit coefficient of friction could lead to improvements in torque tracking. We explored a tether with explicit joints to reduce friction and interference with leg motions, but found that our prototype increased stiffness, damping, and mass compared to the Bowden cable. Prosthesis end-effector mass could be reduced by elimination of the force-amplifying pulley, which appears not to be necessary following tests of maximum Bowden cable tension. Torque could then be measured using a load cell [52] or by instrumenting the toe with strain gauges. We have observed a threefold reduction in torque measurement error using strain gauge sensing in a separate prototype prosthesis endeffector. Improved accuracy was due to the simpler relationship between torque and electrical signal, which was not affected by mechanical elements, such as the chain and sprocket used here, that are difficult to model and may wear over time. Of course, strain gauge sensing is susceptible to electromagnetic interference. Unless an amplifier and A/D converter are mounted to the end-effector, analog wires routed through the tether can lead to increased measurement noise. The mechanical architectures of some end-effectors might also make it inconvenient to incorporate either strain gauges or encoders to measure spring deflection. These factors must be weighed against each other when choosing a torque measurement method. Leaf springs with length-varying cross section could be used to further reduce spring mass. Under higher loads, the Bowden cable itself might exhibit sufficient series compliance, removing the necessity for a spring and allowing incrementally lighter toe structures. We did not carefully quantify the trade-off between torque bandwidth, which favors stiffer springs, and torque disturbance rejection, which favors more compliant springs, and such an analysis could improve performance while adding to the literature on series elastic actuator design. Refinements to the curvature of the passive heel element and toe pad could allow more humanlike center of pressure progression and greater comfort. Improved geometry could also prevent interference between the series leaf springs and the heel section of conventional shoes, perhaps allowing the device to operate within an unmodified shoe. We are presently developing a higher-load prosthesis end-effector that would accommodate larger subjects, a prosthesis with separate inversion-eversion torque control to impact lateral motions as well as the dominant sagittal motions 
addressed here, and an orthosis end-effector as a testbed for rehabilitation techniques. There is much work yet to be done in developing a complete set of high-performance prosthesis and exoskeleton emulators.

We have developed an experimental platform that decouples the challenges of mobile device design from the exploration of human-prosthesis interactions. Our results suggest that platforms of this type will enable rigorous human-subject experiments with the flexibility to evaluate a wide range of parameters and behaviors without laborious tuning of overly specialized devices. This type of technology could become part of a new experimentcentered approach to the development of biomechatronic devices, in which design requirements and trade-offs are established prior to product design tasks such as minimization of mass, envelope, and electricity use. Such an approach could be used to address emerging scientific topics in active prosthetics and orthotics, such as dynamic stability, co-adaptation, and identification of human coordination goals.

\section{Acknowledgment}

The authors thank Soongeun Cha for contributions to mechanical design, Michiel Plooij for contributions to motor and tether design, John Fulmer and James Dillinger for advice on design for manufacture, Gordon Composites for donating leaf springs, Myunghee Kim and Rachel Jackson for contributions to emulator control, and Peter Adamczyk for contributions to the emulator concept. This material is based upon work supported by the National Science Foundation under Grant No. CMMI-1300804.

\section{Appendix A: Tether Characterization}

We performed tests with an anthropomorphic pendulum "leg" to characterize the effects of wearing the instrumented prosthesis while tethered to the off-board motor and controller. We performed 10 trials each for two conditions: tethered, in which both prosthesis and tether were attached normally and maximum ankle torque (and cable tension) were applied, and untethered, in which the prosthesis was attached normally but tether was removed. In each trial, the pendulum was initialized to a starting angle, then allowed to swing freely until an amplitude threshold was crossed (evaluated at the apex of pendulum swing). The trial duration $T$ and number of cycles $n$ were recorded. We modeled the effects of the tether on the pendulum as a rotational stiffness about the hinge $k_{t}$ and a rotational damping component $b_{t}$. The equations of motion for the resulting system, with the small angle approximation, were

$$
I \ddot{\theta}+\left(b_{0}+b_{t}\right) \dot{\theta}+\left(m g c+k_{t}\right) \theta=0
$$

where $I$ is the mass moment of inertia of the pendulum about the hinge, $\theta, \dot{\theta}, \ddot{\theta}$ are the angle, velocity, and acceleration of the pendulum, respectively, $b_{0}$ is the damping coefficient in the untethered condition, $m$ is the pendulum mass, $g$ is the gravitational constant, and $c$ is the distance from the hinge to the center of mass of the pendulum.

Assuming the pendulum is initially at rest $(\dot{\theta}(0)=0)$ and at a prescribed angle $\left(\theta(0)=\theta_{0}\right), \theta$ can be described as a function of time $t$ :

$$
\theta(t)=\theta_{0} e^{-a \cdot t} \cos (\omega t)+\frac{a}{\omega} \theta_{0} e^{-a \cdot t} \sin (\omega t)
$$

Where $a$ is the decay constant and $\omega=2 \pi n T^{-1}$ is the oscillation frequency. Solving for the poles of the system described by Eq. (A1) yields

$$
a=\frac{b_{0}+b_{t}}{2 I}
$$

and

$$
\omega=\sqrt{\frac{m g c+k_{t}}{I}-\frac{\left(b_{0}+b_{t}\right)^{2}}{4 I^{2}}}
$$

Evaluating Eq. (A2) at the moment when the oscillation amplitude reaches the lower threshold $\left(\theta(T)=\theta_{f}\right.$, where $\theta_{f}$ is the angle at the end of the trial, and $\cos (\omega T)=1$ ), and manipulating appropriately, we have the following equation relating damping coefficients to experimental measurements of decay time:

$$
b_{0}+b_{t}=-\frac{2 I}{T} \ln \left(\frac{\theta_{f}}{\theta_{0}}\right)
$$

Substituting Eq. (A5) into Eq. (A4), setting $k_{t}=0$, and rearranging, we have the following relationship between pendulum mass moment of inertia and experimental measurements of decay time and oscillation frequency for the untethered condition:

$$
I=m g c\left(\omega_{u}^{2}+\frac{\ln \left(\frac{\theta_{f}}{\theta_{0}}\right)^{2}}{T^{2}}\right)^{-1}
$$

where $\omega_{u}$ and $T_{u}$ are the frequency and decay period, respectively, for the untethered condition. Finally, solving Eq. (A4), we have the following relationship between tether stiffness and experimental measurements:

$$
k_{t}=I \omega^{2}+\frac{\left(b_{0}+b_{t}\right)^{2}}{4 I}-m g c
$$

We determined $m$ to be $12.0 \mathrm{~kg}$ by weighing the pendulum and $c$ to be $0.34 \mathrm{~m}$ by balancing the pendulum horizontally on a string. From Eq. (A6) we calculated $I$ to be $2.40 \mathrm{Nm}^{2}$. We used Eq. (A5), with $b_{t}=0$, to calculate $b_{0}$ from untethered trials. We then used Eqs. (A5) and (A7) to determine $b_{t}$ and $k_{t}$ from tethered data.

\section{Appendix B: Spring Mass, Material, and Geometry}

The theoretical minimum mass of a spring that meets design requirements for peak load and stiffness (or energy storage) can often be derived in terms of material properties and a geometric constant. We performed calculations for the optimal mass of leaf and coil springs using simple models from classical mechanics. Let us first consider the case of a prismatic member in tension, for which we have the following basic mechanics formulas:

$$
\sigma=\frac{F}{A}=E \varepsilon, \quad \Delta x=l \varepsilon, \quad m=\rho A l
$$

where $\sigma$ is the (uniform) stress in the material, $F$ is the force on the spring, $A$ is the cross-sectional area of the spring, $E$ is the elastic modulus of the material, $\varepsilon$ is the strain of the material, $\Delta x$ is the displacement of the spring, $l$ is the length of the spring, $m$ is the spring mass, and $\rho$ is the density of the material.

In designing a spring, we can set geometric parameters $l$ and $A$ such that when the maximum force $F_{m}$ is applied, a desired maximum displacement $\Delta x_{m}$ and maximum allowable stress $\sigma_{a}$ (typically the failure stress divided by the factor of safety) are simultaneously achieved

$$
A=\frac{F_{m}}{\sigma_{a}}, \quad l=\frac{\Delta x_{m}}{\varepsilon_{m}}=\frac{\Delta x_{m} E}{\sigma_{a}}, \quad \text { and } \quad m=\frac{\rho E}{\sigma_{a}^{2}} F_{m} \Delta x_{m}
$$


This value of $m$ represents the minimum spring mass required to obtain the desired combination of peak load and deflection. Note that this equation for spring mass is independent from geometric parameters. Many spring shapes result in minimum mass relationships of the same form, expressed more generally as

$$
m=2 C_{g} \frac{\rho E}{\sigma_{a}^{2}} U
$$

where $C_{g}$ is a constant arising from spring geometry and $U=(1 / 2) F_{m} \Delta x_{m}$ is the maximum energy stored by the spring. For a prismatic member in tension, $C_{g}=1$. This is the lowest possible value for $C_{g}$, corresponding to the case in which all spring material is maximally strained at peak load. For all other spring shapes, some material will be submaximally strained, and store less energy than possible, but will still contribute equally to spring mass. Other shapes are still useful, however, since the solution to Eq. (B1) often leads to small values for $A$ and large values for $l$, which are inconvenient given practical design constraints.

For leaf springs, we begin with the following equations for bending in a rectangular cantilevered beam:

$$
\sigma_{m}=\frac{M y}{I}, \quad \Delta x=\frac{F l^{3}}{3 E I}, \quad I=\frac{1}{12} b h^{3}, \quad m=\rho b h l
$$

where $\sigma_{m}$ is the maximum stress, $M=F l$ is the moment at the spring support, $y=1 / 2 h$ is the maximum distance from the centroid, $I$ is cross-sectional area moment of inertia, $b$ is spring width, and $h$ is spring height. By eliminating the geometric parameters $b$, $h$, and $l$, we have an equation of the same form as Eq. (B3), with $C_{g}=9$

$$
\begin{aligned}
\sigma_{a} & =\frac{6 M}{b h^{2}} \Rightarrow b=\frac{6 F_{m} l}{\sigma_{a} h^{2}}, \quad \Delta x_{m}=\frac{4 F_{m} l^{3}}{E b h^{3}} \Rightarrow h=\frac{2 l^{2} \sigma_{a}}{3 E \Delta x_{m}}, \quad \text { and } \\
m & =9 \frac{\rho E}{\sigma_{a}^{2}} F_{m} \Delta x_{m}
\end{aligned}
$$

For coil springs, we can model the wire as a rod in torsion

$$
\tau_{m}=\frac{T r}{J}, \quad \Delta \theta=\frac{T l}{G J}, \quad J=\frac{1}{32} \pi r^{4}, \quad m=\rho \pi r^{2} l
$$

where $\tau_{m}$ is the maximum shear stress, $T$ is peak torsion, $r$ is the wire radius, $J$ is cross-sectional polar moment of inertia, $\Delta \theta$ is peak wire rotation, $G$ is the shear modulus, and $l$ is the uncoiled wire length. For steel, we can approximate $\tau_{m} \approx 2 \sigma_{m}$ and $E \approx 8 / 3 G$ [68]. By eliminating the geometric parameters $r$ and $l$, and noting $U=1 / 2 T \Delta \theta$, we have an equation of the same form as Eq. (B3), with $C_{g}=3$

$$
\begin{aligned}
2 \sigma_{a} & =\frac{32 T_{m}}{\pi r^{3}} \Rightarrow r^{3}=\frac{2^{4} T_{m}}{\pi \sigma_{a}}, \\
\Delta \theta_{m} & =\frac{8 \cdot 32 T_{m} l}{3 \pi E r^{4}} \Rightarrow l=\frac{3 \pi E r^{4} \Delta \theta_{m}}{2^{8} T_{m}}, \quad \text { and } \\
m & =\frac{3 \pi^{2} \rho E r^{6} \Delta \theta_{m}}{2^{8} T_{m}}=3 \frac{\rho E}{\sigma_{a}^{2}} T_{m} \Delta \theta_{m}
\end{aligned}
$$

Coil springs can therefore be three times lighter than constant cross-section leaf springs, considering geometric effects alone. However, conventional manufacturing techniques limit the material types that can be formed into useful helical spring shapes.

For any shape that can be expressed in the form of Eq. (B3), the ideal spring material will minimize $\rho E \sigma_{y}^{-2}$. The unidirectional fiberglass material used in the series leaf springs reported here has $\rho=1.9 \times 10^{3} \mathrm{~kg} \mathrm{~m}^{-3}, E=3.8 \times 10^{10} \mathrm{~kg} \mathrm{~m}^{-1} \mathrm{~s}^{-2}$, and $\sigma_{u}$ $=1.1 \times 10^{9} \mathrm{~kg} \mathrm{~m}^{-1} \mathrm{~s}^{-2}[69]$, or $\rho E \sigma_{y}^{-2}=6.4 \times 10^{-5}$. Music wire, the best of conventional spring steels, has $\rho=7.8 \times 10^{3} \mathrm{~kg} \mathrm{~m}^{-3}$,
$E=1.9 \times 10^{11} \mathrm{~kg} \mathrm{~m}^{-1} \mathrm{~s}^{-2}$, and $\sigma_{u}=1.7 \times 10^{9} \mathrm{~kg} \mathrm{~m}^{-1} \mathrm{~s}^{-2}$ (for $r=0.25$ in [68], p. 525), or $\rho E \sigma_{y}^{-2}=5.2 \times 10^{-4}$. Springs optimized for mass and having the same geometry will therefore be about eight times lighter if manufactured from this type of fiberglass than if manufactured from the best spring steel.

\section{References}

[1] Ziegler-Graham, K., MacKenzie, E. J., Ephraim, P. L., Travison, T. G., and Brookmeyer, R., 2008, "Estimating the Prevalence of Limb Loss in the United States: 2005 to 2050," Arch. Phys. Med. Rehab., 89, pp. 422-429.

[2] Ralston, H. J., 1958, "Energy-Speed Relation and Optimal Speed During Level Walking," Int. Z. Angew. Phys., 17, pp. 277-283.

[3] Skinner, H. B., and Effeney, D. J., 1985, "Gait Analysis in Amputees," Am. J. Phys. Med., 64(2), pp. 82-89.

[4] Lehmann, J. F., Price, R., Boswell-Bassette, S., Dralle, A., Questad, K., and DeLateur, B. J., 1993, "Comprehensive Analysis of Energy Storing Prosthetic Feet: Flex Foot and Seattle Foot Versus Standard Sach Foot," Arch. Phys. Med. Rehab., 74, pp. 1225-1231.

[5] Torburn, L., Powers, C. M., Guiterrez, R., and Perry, J., 1995, "Energy Expenditure During Ambulation in Dysvascular and Traumatic Below-Knee Amputees: A Comparison of Five Prosthetic Feet," J. Rehab. Res. Dev., 32, pp. 111-119.

[6] Hoffman, M. D., Sheldahl, L. M., Buley, K. J., and Sandford, P. R., 1997, "Physiological Comparison of Walking Among Bilateral Above-Knee Amputee and Able-Bodied Subjects, and a Model to Account for the Differences in Metabolic Cost," Arch. Phys. Med. Rehab., 78, pp. 385-392.

[7] Waters, R. L., and Mulroy, S., 1999, "The Energy Expenditure of Normal and Pathologic Gait," Gait Posture, 9, pp. 207-231.

[8] Hagberg, K., and Brånemark, R., 2001, "Consequences of Non-Vascular TransFemoral Amputation: A Survey of Quality of Life, Prosthesis Use and Problems," Prosthet. Orthot. Int., 25, pp. 186-194.

[9] Miller, W. C., Speechley, M., and Deathe, A. B., 2002, "Balance Confidence Among People With Lower Limb Amputation," Phys. Therapy, 82, pp. $856-865$.

[10] Hsu, M. J., Nielsen, D. H., Lin-Chan, S. J., and Shurr, D., 2006, "The Effects of Prosthetic Foot Design on Physiologic Measurements, Self-Selected Walking Velocity, and Physical Activity in People With Transtibial Amputation," Arch. Phys. Med. Rehab., 87, pp. 123-129.

[11] Silverman, A. K., Fey, N. P., Portillo, A., Walden, J. G., Bosker, G., and Neptune, R. R., 2008, "Compensatory Mechanisms in Below-Knee Amputee Gait in Response to Increasing Steady-State Walking Speeds," Gait Posture, 28, pp 602-609.

[12] Zidarov, D., Swaine, B., and Gauthier-Gagnon, C., 2009, "Quality of Life of Persons With Lower-Limb Amputation During Rehabilitation and at 3-Month Follow-Up," Arch. Phys. Med. Rehab., 90(4), pp. 634-645.

[13] Morgenroth, D. C., Segal, A. D., Zelik, K. E., Czerniecki, J. M., Klute, G. K. Adamczyk, P. G., Orendurff, M. S., Hahn, M. E., Collins, S. H., and Kuo, A. D., 2012, "The Effect of Prosthetic Foot Push-Off on Mechanical Loading Associated With Knee Osteoarthritis in Lower Extremity Amputees," Gait Posture, 34(4), pp. 502-507.

[14] Au, S. K., Weber, J., and Herr, H., 2007, "Biomechanical Design of a Powered Ankle-Foot Prosthesis," Proceedings of the IEEE International Conference on Rehabilitation Robotics (ICORR), pp. 298-303.

[15] Hitt, J., Oymagil, A. M., Sugar, T., Hollander, K., Boehler, A., and Fleeger, J., 2007, "Dynamically Controlled Ankle-Foot Orthosis (DCO) With Regenerative Kinetics: Incrementally Attaining User Portability," Proceedings of the IEEE International Conference on Robotics and Automation (ICRA), pp. 1541-1546.

[16] Sup, F., Varol, H. A., Mitchell, J., Withrow, T. J., and Goldfarb, M., 2009, "Self-Contained Powered Knee and Ankle Prosthesis: Initial Evaluation on a Transfemoral Amputee," Proceedings of the IEEE International Conference on Rehabilitation Robotics (ICORR), pp. 638-644.

[17] Zelik, K. E., Collins, S. H., Adamczyk, P. G., Segal, A. D., Klute, G. K., Morgenroth, D. C., Hahn, M. E., Orendurff, M. S., Czerniecki, J. M., and Kuo, A. D., 2011, "Systematic Variation of Prosthetic Foot Parameter Affects Center-of-Mass Mechanics and Metabolic Cost During Walking," IEEE Trans. Neural Syst. Rehab. Eng., 19, pp. 411-419.

[18] Cherelle, P., Matthys, A., Grosu, V., Vanderborght, B., and Lefeber, D., 2012 "Mimicking Intact Ankle Behavior With a Powered Transtibial Prosthesis," Proceedings of the Annual International Conference of the IEEE Engineering in Medicine and Biology Society, pp. 544-549.

[19] Segal, A. D., Zelik, K. E., Klute, G. K., Morgenroth, D. C., Hahn, M. E., Orendurff, M. S., Adamczyk, P. G., Collins, S. H., Kuo, A. D., and Czerniecki, J. M., 2012, "The Effects of a Controlled Energy Storage and Return Prototype Prosthetic Foot on Transtibial Amputee Ambulation," Human Movement Sci. 31, pp. 918-931.

[20] Herr, H. M., and Grabowski, A. M., 2012, "Bionic Ankle-Foot Prosthesis Normalizes Walking Gait for Persons With Leg Amputation," Proc. R. Soc. London B, 279, pp. 457-464.

[21] Collins, S. H., and Kuo, A. D., 2010, "Recycling Energy to Restore Impaired Ankle Function During Human Walking," PLoS One, 5, p. e9307.

[22] Madden, J. D., 2007, "Mobile Robots: Motor Challenges and Materials Solutions," Science, 318, pp. 1094-1097.

[23] Anderson, F. C., and Pandy, M. G., 2001, "Dynamic Optimization of Human Walking,” ASME J. Biomech. Eng., 123(5), pp. 381-390. 
[24] Srinivasan, M., and Ruina, A., 2006, "Computer Optimization of a Minimal Biped Model Discovers Walking and Running," Nature, 439(4), pp. 72-75.

[25] Song, S., and Geyer, H., 2012, "Regulating Speed and Generating Large Speed Transitions in a Neuromuscular Human Walking Model," Proceedings of the IEEE International Conference on Robotics and Automation (ICRA), pp. 511-516.

[26] Adamczyk, P. G., Collins, S. H., and Kuo, A. D., 2006, "The Advantages of a Rolling Foot in Human Walking," J. Exp. Biol., 209, pp. 3953-3962.

[27] Fregly, B. J., Besier, T. F., Lloyd, D. G., Delp, S. L., Banks, S. A., Pandy, M. G., and D'Lima, D. D., 2012, "Grand Challenge Competition to Predict in vivo Knee Loads," J. Orthopaed. Res., 30(4), pp. 503-513.

[28] Hirtz, J., Stone, R. B., McAdams, D. A., Szykman, S., and Wood, K. L., 2002, "A Functional Basis for Engineering Design: Reconciling and Evolving Previous Efforts," Res. Eng. Design, 13(2), pp. 65-82.

[29] Pratt, J. E., Krupp, B. T., Morse, C. J., and Collins, S. H., 2004, "The Roboknee: An Exoskeleton for Enhancing Strength and Endurance During Walking," Proceedings of the IEEE International Conference on Robotics and Automation (ICRA), pp. 2430-2435.

[30] Andersen, J. B., and Sinkjær, T., 1995, “An Actuator System for Investigating Electrophysiological and Biomechanical Features Around the Human Ankle Joint During Gait,” IEEE Trans. Rehab. Eng., 3(4), pp. 299-306.

[31] Veneman, J. F., Kruidhof, R., Hekman, E. E. G., Ekkelenkamp, R., van Asseldonk, E. H. F., and van der Kooij, H., 2007, "Design and Evaluation of the LOPES Exoskeleton Robot for Interactive Gait Rehabilitation," IEEE Trans. Neural Syst. Rehab. Eng., 15(3), pp. 379-386.

[32] Sulzer, J. S., Roiz, R. A., Peshkin, M. A., and Patton, J. L., 2009, "A Highly Backdrivable, Lightweight Knee Actuator for Investigating Gait in Stroke," IEEE Trans. Robotics, 25(3), pp. 539-548.

[33] Sawicki, G. S., and Ferris, D. P., 2008, "Mechanics and Energetics of Leve Walking With Powered Ankle Exoskeletons," J. Exp. Biol., 211(9), pp. 1402-1413.

[34] Bruijn, S. M., Meijer, O. G., Beek, P. J., and van Dieën, J. H., 2010, "The Effects of Arm Swing on Human Gait Stability," J. Exp. Biol., 213, pp. 3945-3952.

[35] Flowers, W. C., and Mann, R. W., 1977 “An Electrohydraulic Knee-Torque Controller for a Prosthesis Simulator,” ASME J. Biomech. Eng., 99(1), pp. 3-9.

[36] Abul-Haj, C., and Hogan, N., 1987, "An Emulator System for Developing Improved Elbow-Prosthesis Designs," IEEE Trans. Biomed. Eng., 34(9), pp. 724-737.

[37] Ellis, R. E., Ismaeil, O. M., and Lipsett, M. G., 1996, "Design and Evaluation of a High-Performance Haptic Interface,” Robotica, 4, pp. 321-327.

[38] Hidler, J., Nichols, D., Pelliccio, M., Brady, K., Campbell, D. D., Kahn, J. H. and Hornby, T. G., 2009, "Multicenter Randomized Clinical Trial Evaluating the Effectiveness of the Lokomat in Subacute Stroke," Neurorehab. Neural Repair, 23(1), pp. 5-13.

[39] Griffiths, P. G., Gillespie, R. B., and Freudenberg, J. S., 2011, "A Fundamental Linear Systems Conflict Between Performance and Passivity in Haptic Rendering," IEEE Trans. Robotics, 27(1), pp. 75-88.

[40] Browning, R. C., Modica, J. R., Kram, R., and Goswami, A., 2007, "The Effects of Adding Mass to the Legs on the Energetics and Biomechanics of Walking," Med. Sci. Sports Exercise, 39(3), pp. 515-525.

[41] Gordon, K. E., Sawicki, G. S., and Ferris, D. P., 2006, "Mechanical Performance of Artificial Pneumatic Muscles to Power an Ankle-Foot Orthosis," J. Biomech., 39, pp. 1832-1841.

[42] Whittle, M., 1996, Gait Analysis: An Introduction, Butterworth-Heinemann, Oxford, UK

[43] Pratt, G., and Williamson, M., 1995, "Series Elastic Actuators," Proceedings of the IEEE/RSJ International Conference on Intelligent Robots and Systems (IROS).

[44] Wyeth, G., 2006, "Control Issues for Velocity Sourced Series Elastic Actuators," Proceedings of the Australasian Conference on Robotics and Automation.

[45] Hawes, M. R., and Sovak, D., 1994, "Quantitative Morphology of the Human Foot in a North American Population," Ergonomics, 37(7), pp. 1213-1226.

[46] Winter, D. A., 1990, Biomechanics and Motor Control of Human Movement, 2nd ed., John Wiley and Sons, Toronto, Canada.

[47] Eilenberg, M. F., and Geyer, H., 2010, "Control of a Powered Ankle-Foot Prosthesis Based on a Neuromuscular Model," IEEE Trans. Neural Syst. Rehab. Eng., 18(2), pp. 164-173.
[48] Aoyagi, D., Ichinose, W. E., Harkema, S. J., Reinkensmeyer, D. J., and Bobrow, J. E., 2007, "A Robot and Control Algorithm That Can Synchronously Assist in Naturalistic Motion During Body-Weight-Supported Gait Training Following Neurologic Injury,” IEEE Trans. Neural Syst. Rehab. Eng., 15(3), pp. 387-400.

[49] Caputo, J. M., and Collins, S. H. 2013 “"An Experimental Robotic Testbed for Accelerated Development of Ankle Prostheses," Proceedings of the IEEE International Conference on Robotics and Automation (ICRA), pp. 2630-2635.

[50] Su, J. L., and Dingwell, J. B., 2007, "Dynamic Stability of Passive Dynamic Walking on an Irregular Surface," ASME J. Biomech. Eng., 129(6), pp. 802-810.

[51] Snaterse, M., Ton, R., Kuo, A. D., and Donelan, J. M., 2011, "Distinct Fast and Slow Processes Contribute to the Selection of Preferred Step Frequency During Human Walking," J. Appl. Physiol., 110(6), pp. 1682-1690.

[52] Collins, S. H., and Jackson, R., 2013, "Inducing Self-Selected Human Engagement in Robotic Locomotion Training," Proceedings of the International Conference on Rehabilitation Robotics (ICORR), pp. 1-6.

[53] Roy, A., Krebs, H. I., Williams, D. J., Bever, C. T., Forrester, L. W., Macko, R. M., and Hogan, N., 2009, "Robot-Aided Neurorehabilitation: A Novel Robo for Ankle Rehabilitation," IEEE Trans. Robotics, 25, pp. 569-582.

[54] Burse, R. L., and Pandolf, K. B., 1979, "Physical Conditioning of Sedentary Young Men With Ankle Weights During Working Hours," Ergonomics, 22, pp. 69-78.

[55] Hitt, J., Sugar, T., Holgate, M., Bellman, R., and Hollander, K., 2009, "Robotic Transtibital Prosthesis With Biomechanical Energy Regeneration," Ind. Robot Int. J., 36(5), pp. 441-447.

[56] Bawa, P., and Stein, R. B., 1976, "Frequency Response of Human Soleus Muscle," J. Neurophysiol., 39(4), pp. 788-793.

[57] Agarwal, G. C., and Gottlieb, G. L., 1977, "Oscillation of the Human Ankle Joint in Response to Applied Sinusoidal Torque on the Foot," J. Physiol., 268, pp. $151-176$.

[58] Noël, M., Cantin, B., Lambert, S., Gosselin, C. M., and Bouyer, L. J., 2008 , "An Electrohydraulic Actuated Ankle Foot Orthosis to Generate Force Fields and to Test Proprioceptive Reflexes During Human Walking," IEEE Trans. Neural Syst. Rehab. Eng., 16, pp. 390-399.

[59] Stienen, A. H. A., Hekman, E. E. G., ter Braak, H., Aalsma, A. M. M., van der Helm, F. C. T., and van der Kooij, H., 2010, "Design of a Rotational Hydroelastic Actuator for a Powered Exoskeleton for Upper Limb Rehabilitation," IEEE Trans. Biomed. Eng., 57, pp. 728-735.

[60] Winter, D. A. 1991, The Biomechanics and Motor Control of Human Gait: Normal, Elderly and Pathological, Waterloo Biomechanics, Waterloo, Canada.

[61] Pratt, J., Krupp, B., and Morse, C., 2002, "Series Elastic Actuators for High Fidelity Force Control," Ind. Robot Int. J., 29, pp. 234-241.

[62] Schiele, A., Letier, P., van der Linde, R., and van der Helm, F., 2006, "Bowden Cable Actuator for Force-Feedback Exoskeletons," Proceedings of the IEEE/ RSJ International Conference on Intelligent Robots and Systems (IROS), pp. 3599-3604.

[63] Zoss, A., Kazerooni, H., and Chu, A., 2005, "On the Mechanical Design of the Berkeley Lower Extremity Exoskeleton (BLEEX),” Proceedings of the IEEE/ RSJ International Conference on Intelligent Robots and Systems (IROS), pp. $3465-3472$

[64] Stephens, B. J., and Atkeson, C. G., 2010, "Dynamic Balance Force Control for Compliant Humanoid Robots," Proceedings of the IEEE/RSJ International Conference on Intelligent Robots and Systems (IROS), pp. 1248-1255.

[65] Versluys, R. Deckers, K. Van Damme, M., Van Ham, R. Steenackers, G., Guillaume, P., and Lefeber, D., 2009, "A Study on the Bandwidth Characteristics of Pleated Pneumatic Artificial Muscles," Appl. Bionics Biomech., 6(1) pp. 3-9.

[66] Versluys, R., Desomer, A., Lenaerts, G., Pareit, O., Vanderborght, B., Perre, G. Peeraer, L., and Lefeber, D., 2009, "A Biomechatronical Transtibial Prosthesis Powered by Pleated Pneumatic Artificial Muscles," Int. J. Model. Ident. Control, 4(4), pp. 394-405.

[67] Schiele, A., 2008, "Performance Difference of Bowden Cable Relocated and Non-Relocated Master Actuators in Virtual Environment Applications," Proceedings of the IEEE/RSJ International Conference on Intelligent Robots and Systems (IROS), pp. 3507-3512.

[68] Budynas, R. G., and Nisbett, J. K., 2011, Shigley's Mechanical Engineering Design, 9th ed. McGraw-Hill, New York.

[69] Gordon Composites, Inc., 2012, GC-67-UB: Unidirectional Fiberglass Bar Stock, August 2012, URL: http://www.gordoncomposites.com/. 